УДК 539.37

\title{
Strong Bending of a Beam from a Fibrous Composite, Differently Resistant to Tension and Compression
}

\author{
Boris D. Annin* \\ Lavrentyev Institute of Hydrodynamics RAS SB \\ Lavrentyeva, 15, Novosibirsk, 630090 \\ Russia \\ Vladimir M. Sadovskii ${ }^{\dagger}$ \\ Institute of Computational Modelling RAS SB \\ Akademgorodok, 50/44 Krasnoyarsk, 660036 \\ Russia \\ Igor E. Petrakov $\ddagger$ \\ Institute of Mathematics and Computer Science \\ Siberian Federal University \\ Svobodny, 79, Krasnoyarsk, 660041 \\ Russia \\ Anton Yu. Vlasov ${ }^{\S}$ \\ Reshetnev Siberian State University of Science and Technology \\ Semafornaya, 433/1, Krasnoyarsk, 660059 \\ Russia
}

Received 13.03.2019, received in revised form 04.04.2019, accepted 20.06.2019

For the analysis of bending of a thin rod made of fiber composite, the generalized Euler elastic equation is used, taking into account different resistance of the material to tension and compression, the influence of transverse shear, elongation of the axis and independent rotations of the reinforcing elements relative to the matrix. Based on Newton's method, a computational algorithm has been developed for solving the static bending problem. A method for determining phenomenological parameters of the composite has been implemented, including photographing the bending state of the rod under the action of a system of forces and couple forces, digital processing of the photography and solving the inverse coefficient problem. The method was validated by comparing the results of computations with a laboratory physical experiment. It is shown that the moduli of elasticity in tension and compression of carbon fiber composite used in the experiment, essentially differ, and that the use of equal moduli in determining bending stiffness results in a significant error in the deflection calculations.

Keywords: fiber reinforced composite, bending state, different resistance to tension and compression, axial deformation, Tymoshenko effect, Cosserat effect.

DOI: 10.17516/1997-1397-2019-12-5-533-542.

\footnotetext{
*annin@hydro.nsc.ru

${ }^{\dagger}$ sadov@icm.krasn.ru

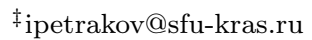

$\S$ vlasov.anton@gmail.com

(C) Siberian Federal University. All rights reserved
} 


\section{Introduction}

Designing and creating composite materials with predetermined properties for modern engineering applications are directly related to the development of appropriate mathematical models $[1,2]$ and methods for studying them [3,4]. Analysis of the experimental data of the deformation of composites reinforced with fibers in one or several directions shows that such materials, as a rule, have different elastic moduli for tension and compression, and different limits of tensile and compressive strength [5,6]. Ice reinforced with geosynthetic fibers is a composite of this type. Fibers strongly harden the material under tension and have almost no effect on its mechanical characteristics during compression. Another example is organoplastics with aramid fibers, whose strength is 5 to 10 times lower in compression than in tension as shown by experiments. Many other types of composite materials have different resistance to tension and compression, in particular, fiberglass and carbon fiber epoxy-based composites.

An example of a stress-strain state with different resistance of a fiber composite to tension and compression is the state of pure bending. When a plate or rod bends, the upper fibers in the direction of the deflection stretch, and the lower ones contracts. Reinforcement has smaller effect in the zone of contraction than in the stretching zone. It is reduced to zero when bending reinforced ice. In the case of high-strength carbon-reinforced plastics, when the curvature increases in the zone of contraction the stability is lost, the fibers are destroyed or detached from the matrix and, as a result, the reinforcement effect is reduced. Another reason for the different resistance of the composite to tension and compression can be the difference in moduli of the matrix of a high-molecular polymer, which is more stiff under tension than during compression due to intermolecular bonds of polymer chains, or more pliable due to the porosity of the polydisperse microstructure. The position of the neutral line in pure bending of a plate (rod) of a material with different tensile and compressive strength does not coincide with the median line of the classical problem of bending with a symmetric diagram of uniaxial tension-compression. Analysis of the exact solution of the bending problem shows that the error in determining the deflection of the composite plate can reach $90 \%$ if it is assumed in the calculations that the elastic modulus does not change when the sign of the deformation changes. Therefore, different resistances to tension and compression must be taken into account when constructing constitutive equations for solving the boundary problems of the dynamics and statics of structural elements made of fibrous composites.

The ultimate goal of the approach suggested by the authors is to develop universal computational methods and algorithms for solving boundary problems of deformation and fracture of composite structures under alternating loads, when the distribution of tension and compression zones is unknown and varies with time. This approach was used in the problems of mechanics of different strength, porous and bulk media [7]. In this paper, we implemented a method for determining the elastic parameters of a composite material based on solving inverse coefficient problems for measuring the finite deflections of a cantilever rod.

\section{The equation of the elastic line}

The classical Euler elastic equation describing the plane bending of a thin flexible rod of rectangular cross section with arbitrary deflection can be obtained on the basis of the Lagrange variational principle, according to which the minimum energy integral is reached at the equilibrium state of the rod 


$$
J(x, y, \theta)=\int_{0}^{l}\left(\frac{1}{2} D(s)\left|\theta^{\prime}\right|^{2}-\mu g y\right) d s-F_{x} x(l)-F_{y} y(l)-M \theta(l),
$$

where $l$ is the length of rod; $\mu$ is the linear weight; $g$ is the acceleration of gravity; $F_{x}$ and $F_{y}$ are the projection of the vector of bending force applied to the right end: $F_{x}=F \cos \alpha, F_{y}=F \sin \alpha$, $M$ is the bending moment; $D$ is the bending stiffness, depending on the length $s$; prime means $s$ derivative. For simplicity, the left end of the rod is considered fixed. The angle of inclination of the rod axis $\theta(s)$ and the coordinates of a point on the axis $x(s), y(s)$ are not independent functions. They satisfy the geometric constraints:

$$
x^{\prime}=\cos \theta, \quad y^{\prime}=\sin \theta .
$$

The boundary conditions of rigid fixation at the left end of the rod are formulated as: $\theta(0)=$ $=x(0)=y(0)=0$. These are the main conditions for a variational problem that must be satisfied by variable functions. The natural boundary conditions at the right end are automatically obtained by minimizing the integral.

The mathematical model under consideration is based on the assumption that with a strong bend of the rod, the angles of rotation of linear elements are finite and the deformations are small, therefore, according to Hooke's law, the bending moment is proportional to the curvature: $m=D æ\left(æ=\theta^{\prime}\right)$. Thus, the variational principle (1) corresponds to a physically linear, geometrically non-linear model of flexing a flexible rod.

The conditional minimization problem (1), (2) is equivalent to the unconditional minimum Lagrangian problem

$$
L(x, y, \theta)=J(x, y, \theta)-\int_{0}^{l} \lambda_{x}\left(x^{\prime}-\cos \theta\right) d s-\int_{0}^{l} \lambda_{y}\left(y^{\prime}-\sin \theta\right) d s
$$

where $\lambda_{x}(s), \lambda_{y}(s)$ are Lagrange multipliers corresponding to constraints (2). Varying the functional (3) with three independent arguments, we get the equations

$$
\left(D(s) \theta^{\prime}\right)^{\prime}=-\lambda_{x} \sin \theta+\lambda_{y} \cos \theta, \quad \lambda_{x}^{\prime}=0, \quad \lambda_{y}^{\prime}=\mu g
$$

and boundary conditions at the right end:

$$
D(l) \theta^{\prime}(l)=M, \quad \lambda_{x}(l)=-F_{x}, \quad \lambda_{y}(l)=-F_{y} .
$$

From this it follows that the angle of inclination of the rod axis (elastic line) is a solution to a boundary value problem for a second-order semi-linear equation

$$
\left(D(s) \theta^{\prime}\right)^{\prime}=F \sin (\theta-\alpha)+\mu g(s-l) \cos \theta, \quad \theta(0)=0, \quad D(l) \theta^{\prime}(l)=M .
$$

The shape of the elastic line is determined by solving the problem (3) using the equations (2) taking into account the boundary conditions at the left end.

The obtained equation with an arbitrary direction of force at the end of the rod is derived, for example, in $[1,8]$. In this case, unlike its usual use, the fiber composite rod is multi-modular: Young's modulus under tension $E^{+}$significantly greater than the modulus under compression $E^{-}$. Such difference is due to local loss of stability and partial destruction of high-strength fibers in a flexible matrix, which are in a state of axial compression during bending of the rod. With a further increase in curvature, a loss of stability is followed by a stage of destruction of the matrix with fiber delamination. This leads to a further decrease in bending stiffness, and the process becomes physically nonlinear. 


\section{Accounting for axial deformation and transverse shear}

In order to take into account the longitudinal deformation of the elastic line and the influence of transverse shears, the vector of the position of the point of the deformed rod will be represented as a linear decomposition in thickness:

$$
X(s, t)=x(s)+t \psi_{x}(s), \quad Y(s, t)=y(s)+t \psi_{y}(s),
$$

where $s, t$ are Lagrangian coordinates of a point relative to the Cartesian coordinate system associated with the central axis of the rod. According to the Cayley polar decomposition theorem, the distortion tensor on the axis

$$
Q=\left(\begin{array}{ll}
x^{\prime} & \psi_{x} \\
y^{\prime} & \psi_{y}
\end{array}\right)
$$

is equal to the product of the orthogonal tensor $R$, which describes the rotation of a neighborhood of a point on the axis, and the symmetric tensor, the Cauchy strain measure:

$$
Q=R \cdot U, \quad R=\left(\begin{array}{cc}
\cos \theta & -\sin \theta \\
\sin \theta & \cos \theta
\end{array}\right), \quad U=\left(\begin{array}{cc}
1+\varepsilon & \gamma \\
\gamma & 1-\nu \varepsilon
\end{array}\right) .
$$

In this decomposition $\varepsilon$ is a longitudinal deformation, $\gamma$ is a shear deformation. By assumption, there are no external forces on the side surface of the rod, therefore the thickness compression strain is equal to $-\nu \varepsilon$, where $\nu$ is the Poisson's ratio. The deformation of the compression across the plane of the bend is also equal to $-\nu \varepsilon$.

Polar decomposition allows to obtain dependencies, generalizing restrictions (2):

$$
x^{\prime}=(1+\varepsilon) \cos \theta-\gamma \sin \theta, \quad y^{\prime}=(1+\varepsilon) \sin \theta+\gamma \cos \theta .
$$

In addition, it also follows the formulas

$$
\psi_{x}=\gamma \cos \theta-(1-\nu \varepsilon) \sin \theta, \quad \psi_{y}=\gamma \sin \theta+(1-\nu \varepsilon) \cos \theta,
$$

that are used to determine the position of a point of the rod, separated from the axis.

The energy integral with respect to axial deformation and transverse shear takes the form

$$
J(x, y, \theta)=\frac{1}{2} \int_{0}^{l}\left(A \varepsilon^{2}+B \gamma^{2}+D\left|\theta^{\prime}\right|^{2}-2 \mu g y\right) d s-F_{x} x(l)-F_{y} y(l)-M \theta(l),
$$

where $A=\left(1+2 \nu^{2}\right) E S, B=G S, E$ is the Young's modulus, $G$ is the shear modulus, $S$ is the rod cross-sectional area. Moving from the problem of conditional minimization (4), (5) of the problem of the unconditional minimum of the corresponding Lagrangian, as a result of the variation we obtain the following equations for the deformations

$$
A \varepsilon=F \cos (\theta-\alpha), \quad B \gamma=-F \sin (\theta-\alpha)
$$

and the boundary problem for the equation of the elastic line

$$
\left(D(s) \theta^{\prime}\right)^{\prime}=F \sin (\theta-\alpha)-a F \sin 2(\theta-\alpha)+\mu g(s-l) \cos \theta
$$

with boundary conditions given in (3). It follows from the equation (6) that the influence of transverse shear and axial deformation can be neglected if the dimensionless parameter (which is a function if the rod has a variable cross section, or if the elastic moduli depend on $s$ ) in the right-hand side

$$
a=\frac{F}{2}\left(\frac{1}{B}-\frac{1}{A}\right)
$$

is much lower than one. If $a$ is about a one, then this approximation is invalid. 


\section{Cosserat effect}

Cosserat's theory of elasticity takes into account the independent rotation of the particles of the microstructure of the material (reinforcing elements in the case of fiber composite) relative to the deformable medium-the matrix. In the physically linear approximation, the moment of resistance to rotation of a particle is proportional to the angle of relative rotation $\varphi$. The rotation tensor $R$ is equal to the superposition of the relative $R_{r}$ and transferring $R_{e}$ rotations: $R=R_{e} \cdot R_{r}$. In the plane case

$$
R_{r}=\left(\begin{array}{cc}
\cos \varphi & -\sin \varphi \\
\sin \varphi & \cos \varphi
\end{array}\right), \quad R_{e}=\left(\begin{array}{cc}
\cos (\theta-\varphi) & -\sin (\theta-\varphi) \\
\sin (\theta-\varphi) & \cos (\theta-\varphi)
\end{array}\right) .
$$

The polar decomposition of the distortion tensor contains only the transferring rotation tensor: $Q=R_{e} \cdot U$. A measure of the deformation of a structurally inhomogeneous medium is an asymmetric tensor $\Lambda=R_{r}^{*} \cdot U$ (asterisk means transpose), which simultaneously characterizes the deformation of the elements of the medium and relative particle rotations (see [9]). From the equation $\Lambda=R^{*} \cdot Q$ follows that it is equal to the unit tensor in the case of rotation of the medium as a rigid unit, when $Q=R$. For thin rod

$$
\begin{aligned}
\Lambda & =\left(\begin{array}{cc}
\cos \varphi & \sin \varphi \\
-\sin \varphi & \cos \varphi
\end{array}\right) \cdot\left(\begin{array}{cc}
1+\varepsilon & \gamma \\
\gamma & 1-\nu \varepsilon
\end{array}\right), \\
\left(\begin{array}{ll}
x^{\prime} & \psi_{x} \\
y^{\prime} & \psi_{y}
\end{array}\right) & =\left(\begin{array}{cc}
\cos (\theta-\varphi) & -\sin (\theta-\varphi) \\
\sin (\theta-\varphi) & \cos (\theta-\varphi)
\end{array}\right) \cdot\left(\begin{array}{cc}
1+\varepsilon & \gamma \\
\gamma & 1-\nu \varepsilon
\end{array}\right) .
\end{aligned}
$$

The last equation for the distortion tensor is followed by dependencies that generalize (4):

$$
x^{\prime}=(1+\varepsilon) \cos (\theta-\varphi)-\gamma \sin (\theta-\varphi), \quad y^{\prime}=(1+\varepsilon) \sin (\theta-\varphi)+\gamma \cos (\theta-\varphi),
$$

and formulas for $\psi_{x}$ и $\psi_{y}$ :

$$
\psi_{x}=\gamma \cos (\theta-\varphi)-(1-\nu \varepsilon) \sin (\theta-\varphi), \quad \psi_{y}=\gamma \sin (\theta-\varphi)+(1-\nu \varepsilon) \cos (\theta-\varphi)
$$

The equation for $\Lambda$ shows that the invariants of the deformed state are $\varepsilon, \gamma$ и $\varphi$. Therefore, the elastic energy integral is written as:

$$
J(x, y, \theta)=\frac{1}{2} \int_{0}^{l}\left(A \varepsilon^{2}+B \gamma^{2}+C \varphi^{2}+D\left|\theta^{\prime}\right|^{2}-2 \mu g y\right) d s-F_{x} x(l)-F_{y} y(l)-M \theta(l),
$$

where $C=K S, K$ is Cosseur Modulus.

The use of the Lagrange formalism to the problem of conditional minimization (7), (8) with the subsequent calculation of the variation allows to obtain equations for axial deformation, shear and relative rotation angle:

$A \varepsilon=F \cos (\theta-\varphi-\alpha), \quad B \gamma=-F \sin (\theta-\varphi-\alpha), \quad C \varphi=F \sin (\theta-\varphi-\alpha)-a F \sin 2(\theta-\varphi-\alpha)$.

The refined equation of the elastic line takes the form:

$$
\left(D \theta^{\prime}\right)^{\prime}=C \varphi+\mu g(s-l) \cos \theta
$$

The boundary conditions for this equation do not change, remaining as in (3). 
According to the implicit function theorem, a nonlinear equation for $\varphi$ determines a unique and continuously differentiable dependence $\varphi=\varphi(\theta)$, at least if the condition $(1+2 a) F<C$ is met. In this case, the implicit function corresponding to the equation is strictly monotonic in the variable $\varphi$ and besides $\varphi=0$ when $\theta=\alpha$. It is possible that if this condition is violated, a specific loss of stability of the rod may occur due to the instability of the spatial orientation of the reinforcing elements. Without discussing the issue of stability in this article, we assume that the angle of relative rotation $\varphi$ is infinitely small. Then we have the linearized equation, the solution of which is in a closed form:

$$
\varphi=b \frac{\sin (\theta-\alpha)-a \sin 2(\theta-\alpha)}{1+b \cos (\theta-\alpha)-2 a b \cos 2(\theta-\alpha)},
$$

where $b=F / C$ is the one more dimensionless parameter (dimensionless function in the case of a non-uniform rod).

\section{Numerical results}

In the numerical solution of the boundary problem for equation (9) with an arbitrary, but specified distribution of bending stiffness and elastic moduli, a second-order difference scheme was used.

The rod was divided along the axis by a uniform grid $s_{j}=(j+1 / 2) \Delta s$ consisting of $n$ nodes with a step $\Delta s=l /(n-0.5)$. The nonlinear right-hand side of the equation was taken into account iteratively using the Newton method. As an initial approximation, a non-flexural state of the rod with nodal inclination angle $\theta_{j}=0$ or a bending state with a linear distribution of the angle along the axis of the rod was taken. The new approximation $\theta^{j}$ was determined using the previous one $\theta_{j}$ from the system of linearized equations $(j=1, \ldots, n-1)$ :

$$
\begin{gathered}
D_{j+1 / 2}\left(\theta^{j+1}-\theta^{j}\right)-D_{j-1 / 2}\left(\theta^{j}-\theta^{j-1}\right)=C_{j}\left(\varphi\left(\theta_{j}\right)+\left(\theta^{j}-\theta_{j}\right) \varphi_{1}\left(\theta_{j}\right)\right) d s^{2}+ \\
+\mu g\left(s_{j}-l\right)\left(\cos \theta_{j}-\left(\theta^{j}-\theta_{j}\right) \sin \theta_{j}\right) d s^{2}
\end{gathered}
$$

where $\varphi_{1}$ is the first derivative $\varphi$ with respect to $\theta$. In conjunction with the boundary conditions

$$
\theta^{0}=0, \quad D_{n+1 / 2}\left(\theta^{n+1}-\theta^{n}\right)=M \Delta s
$$

this system was solved by the tridiagonal matrix algorithm. To finish the iteration process the condition $\max \left|\theta^{j}-\theta_{j}\right| \leqslant \varepsilon \max \left|\theta^{j}\right|$ was used, in which $\varepsilon$ was the predetermined computation error.

Based on the developed computer program, a series of calculations were carried out in the Matlab system to determine the Young's modulus of the composite material under compression. Previously, a similar technique was used in the work [10].

For a thin rod of rectangular cross section $S=w h$ of carbon-fiber plastic with a volume fraction of fibers of about $50 \%$, whose height was $h=1.16 \mathrm{~mm}$, width $w=2.86 \mathrm{~mm}$, Young's modulus was measured upon uniaxial tension by using experimental methods, a reliable value of which was $114 \mathrm{GPa}$. Next a plane bending of the rod with a length $l=280 \mathrm{~mm}$ was produced using the force $F=1.2 \mathrm{~N}$ acting on the rod ends as shown in Fig. 1. Rod linear weight is equal to $\mu=4.7 \mathrm{~g} / \mathrm{m}$. The corresponding gravity can be ignored, since the deflection of the end of the rod due to its own weight increases by about $0.2 \%$ of the deflection in the described experiment. 


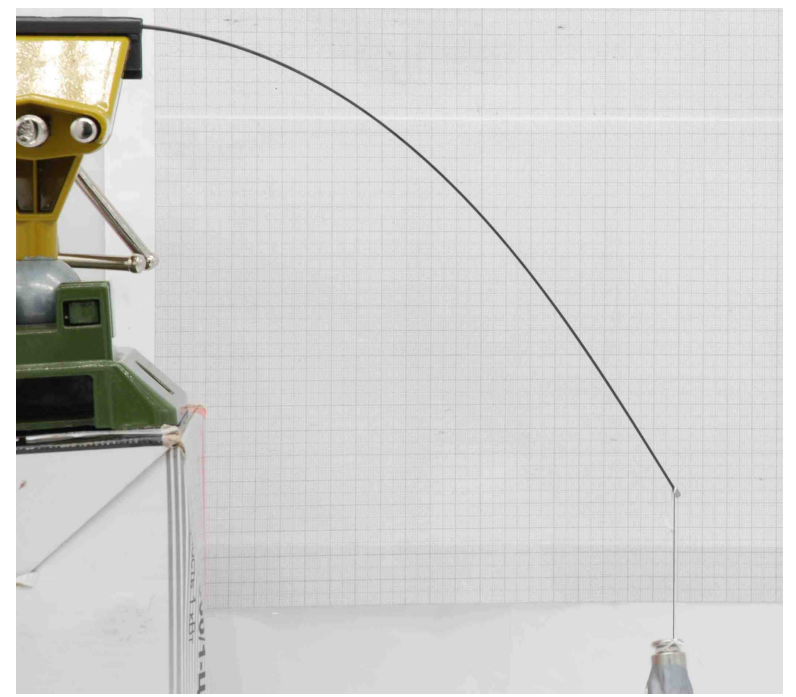

Fig. 1. Plane bending of thin flexible rod

Photos of the bended rod were processed to obtain a plane projection, then digitized. The data on deflection along the length of the rod were used to solve the inverse problem of determining the flexural rigidity $D$ (stiffness was considered constant) using the least squares method. Using the built-in functions of Matlab, the problem of minimizing the standard deviation was solved:

$$
\delta^{2}=\sum_{k=1}^{m}\left|y\left(x_{k}\right)-\bar{y}_{k}\right|^{2} \rightarrow \min _{D} !
$$

Here $y\left(x_{k}\right)$ - the calculated values of the deflection of the rod in a given system of points along the horizontal axis $x_{1}, x_{2}, \ldots, x_{m} ; \bar{y}_{k}$ - measured (digitized) deflection values at the same points (Fig. 2). According to calculations, the bending stiffness minimizing the standard deviation in a system of $m=30$ equidistant points for the rod under consideration is equal to $D=0.0288 \mathrm{~N} \cdot \mathrm{m}^{2}$. The elastic line graph is depicted in comparison with the measurement results in Fig. 3.

When calculating the bending $\varepsilon=10^{-7}$ was used. To achieve this accuracy, 5 iterations of the Newton method were required.

To check the reliability of the obtained values of bending stiffness, a large series of experiments on bending of shorter and longer rods from the same composite from $l=100 \mathrm{~mm}$ to $l=400 \mathrm{~mm}$ was performed. The difference between the experimental and calculated deflections of the rod end is about $\pm 3 \%$ of the calculated value with an unpredictable sign change, which points to measurement errors rather than to the imperfection of the computational model.

In the theory of bending of multi-modulus rods, bending stiffness and Young's moduli for tension $E^{+}$and compression $E^{-}$are related by the following equations:

$$
h_{+}=\frac{\sqrt{E^{-}} h}{\sqrt{E^{-}}+\sqrt{E^{+}}}, \quad h_{-}=\frac{\sqrt{E^{+}} h}{\sqrt{E^{-}}+\sqrt{E^{+}}}, \quad D=a\left(\frac{E^{+} h_{+}^{3}}{3}+\frac{E^{-} h_{-}^{3}}{3}\right),
$$

where $h_{+}$is the height of the stretchable part of the rod section, $h_{-}$is the height of the compressible part. From equations (10) using already found $D$ and $E^{+}$the value $E^{-}=56.8 G P a$ was obtained, which is $49.8 \%$ of $E^{+}$. 


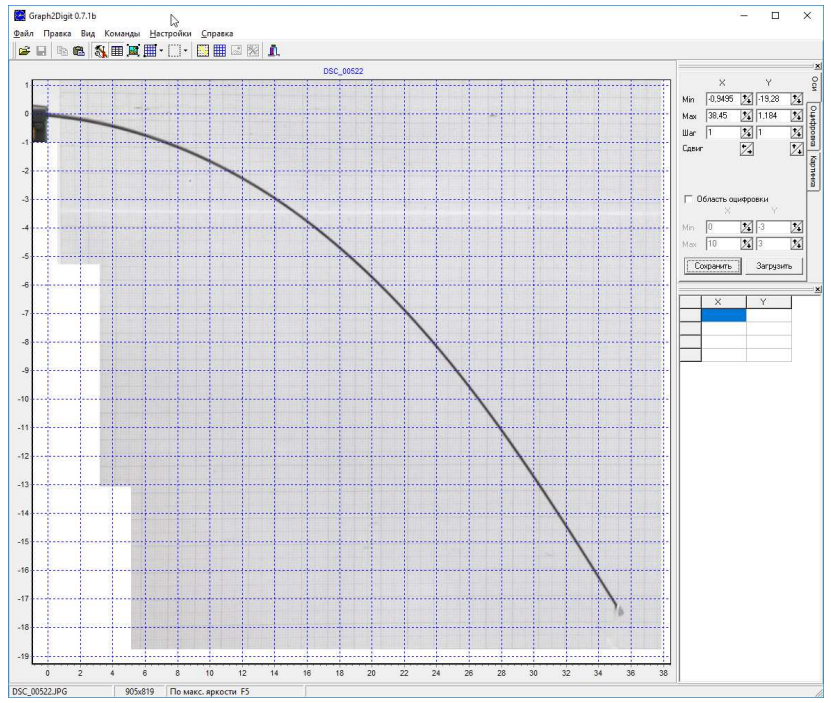

Fig. 2. Digitization photos

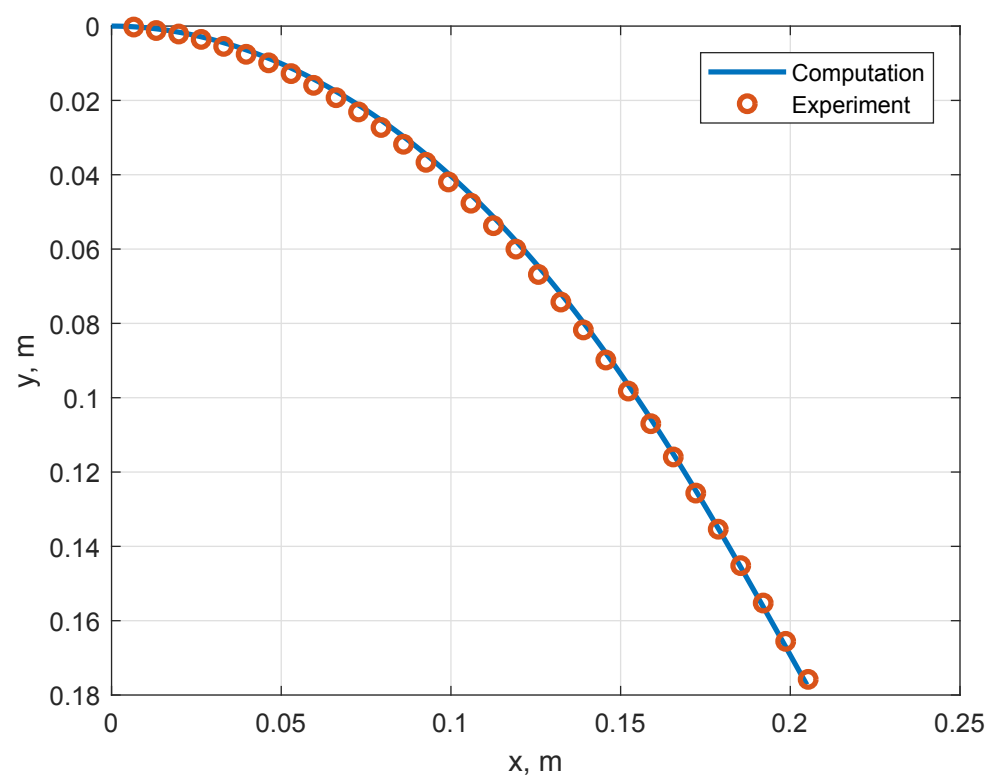

Fig. 3. Rod bending state

Thus, the composite material under consideration is significantly multi-modular. Additional calculations showed that if we assume that the Young's modulus for compression is equal to the experimental modulus for tensile, this leads to an underestimate of the deflection. The value of the relative error in this case will be $16 \%$. If in the experiment to use a thicker rod with $h=1.74 \mathrm{~mm}$, then the error will be equal to $28 \%$, if $h=2.32 \mathrm{~mm}$, then the error increases to $31 \%$ in relation to the deflection. 


\section{Conclusion}

The analysis of the numerical experimental results in the problem of the strong rod bending showed that taking into account different resistances of the fiber composite (carbon fiber) to tension and compression is of fundamental importance. This follows from the comparison of the numerical results with the results of experimental measurements with the simplest cantilever bending. According to the calculations performed, with this method of loading, the influence of other factors considered above (axial deformation, shear and relative rotations) turns out to be negligible. The problem of choosing loading methods, in which the Tymoshenko effect associated with shifts, and the Cosserat effect, which characterizes the contribution of rotation of reinforcing elements relative to the matrix, to identify the corresponding coefficients of the refined bending equation, are beyond the scope of this article, thus remaining open.

The work was supported by the Russian Foundation for Basic Research (grant no. 19-0100511) and by the Ministry of Education and Science of the Russian Federation (contract no. 02.G25.31.0147). The presented calculations were performed on the Flagman computer of the Institute of Computational Modeling of the SB RAS.

\section{References}

[1] Y.N.Rabotnov, Mechanics of a deformable solid, Moscow, Nauka, 1988 (in Russian).

[2] R.M.Christensen, Mechanics of composite materials, Dover Publications, New York, 2005.

[3] B.E.Pobedrya, Mechanics of composite materials, Moscow, Izdatelstvovo Mosk. un-ta, 1984 (in Russian).

[4] A.S.Kravchuk, V.P.Mayboroda, Y.S.Urzhumtsev, Mechanics of polymer and composite materials: experimental and numerical methods, Moscow, Nauka, 1985 (in Russian).

[5] N.M.Matchenko, L.A.Tolokonnikov, Relations between stresses and strains for multimodular elastic media, Izv. AN SSSR. Ser. "Mekhanika tverdogo tela", (1968), no. 6, 108-110 (in Russian),

[6] A.I.Oleinikov, Evaluation of stiffness and strength of laminated composites, Kompozity $i$ nanostruktury, 9(2017), no. 2, 77-79 (in Russian).

[7] O.V.Sadovskaya, V.M.Sadovskii, Mathematical modeling in mechanics of granular materials, Advanced Structured Materials, Springer, Heidelberg, New York, Dordrecht, London, Vol. 21, 2012.

[8] E.P.Popov, Theory and calculation of flexible elastic rods, Moscow, Nauka, 1986 (in Russian).

[9] V.M.Sadovskii, Thermodynamically consistent equations of the couple stress elasticity, Dal'nevostochnyj matematicheskij zhurnal, 16(2016), no. 2, 209-222 (in Russian).

[10] R.A.Kayumov, S.A.Lukankin, V.N.Pajmushin, S.A.Holmogorov, Identification of the mechanical characteristics of fiber-reinforced composites, Uchen. zap. Kazan. un-ta. Ser. Fiz.matem. nauki, 157(2015), no. 4, 112-132 (in Russian). 


\section{Сильный изгиб стержня из волокнистого композита, по-разному сопротивляющегося растяжению и сжатию}

Борис Д. Аннин

Институт гидродинамики им. Лаврентьева СО РАН Лаврентьева, 15, Новосибирск, 630090

Россия

Владимир М. Садовский

Институт вычислительного моделирования СО РАН Академгородок, 50/44, Красноярск, 660036

Россия

Игорь Е. Петраков

Институт математики и фундаментальной информатики

Сибирский федеральный университет Свободный, 79, Красноярск, 660041

Россия

Антон Ю. Власов

Сибирский государственный университет науки и технологий Семафорная, 433/1, Красноярск, 660059

Россия

К анализу изгиба тонкого стержня из волокнистого композита применяется обобшенное уравнение эластики Эйлера, учитывающее разное сопротивление материала растяжению и сжатию, влияние поперечных сдвигов, удлинение вдоль оси и независимые повороты армируюших элементов относительно матриць. На основе метода Нъютона разработан вычислителъный алгоритм для решения статической задачи изгиба. Реализована методика определения феноменологических параметров композита, включающая в себя фотосвемку изгибного состояния стержня под действием системы сил и моментов сил, иифровую обработку ботографии и решение обратной коэфбициентной задачи. Выполнена валидация методики путем сравнения результатов расчетов с лабораторным физическим экспериментом. Показано, что для углепластика, применяемого в эксперименте, модули упругости при растяжении и при сжатии существенно различаются, и что использование равных модулей при определении жесткости на изгиб приводит к систематической ошибке в расчетах прогиба.

Ключевые слова: волокнистый композит, изгибное состояние, разное сопротивление растяжению и сжстию, осевая деформация, эбфект Тимошенко, эбфект Коссера. 